\title{
A Transição de Serviços de Saúde de Crianças e Jovens para Serviços de Saúde de Adultos: Uma Realidade com Impacto no Prognóstico
}

\section{The Transition of Children and Adolescent Healthcare Services to Adult Healthcare Services: A Reality with Impact on Prognosis}

José SALGADO $\triangle^{1}$

Acta Med Port 2022 Mar;35(3):161-163 - https://doi.org/10.20344/amp.15912

Palavras-chave: Acesso aos Serviços de Saúde; Adolescente; Criança; Encaminhamento e Consulta

Keywords: Adolescent; Child; Health Services Accessibility; Transition to Adult Care

Aos 18 anos, as pessoas com patologias de evolução prolongada mudam do serviço que os acompanhavam, por vezes desde há muito tempo, para um novo serviço. Esta mudança ou transição, parecendo trivial, tem um impacto significativo no curso da doença, sendo importante haver um plano consistente para a fazer. ${ }^{1}$

Todavia, constata-se que uma boa transição é rara mesmo em sistemas de saúde bem organizados, dado que este processo deve ser intencional e planeado, considerando as necessidades médicas, psicossociais e educacionais / vocacionais de adolescentes e jovens adultos com doenças crónicas de qualquer tipo.

Este processo torna-se complexo porque envolve geralmente jovens com doenças crónicas que sabem que a doença fará parte das suas vidas para sempre. ${ }^{2}$

Podem assim reagir com raiva, tristeza ou negação, embora a maioria aprenda a conviver com isso, se o processo for conduzido adequadamente.

Nos Estados Unidos, cerca de 750000 jovens com "necessidades de saúde especiais" distintas, (por exemplo, diabetes, transplantes, doenças cardíacas, psiquiátricas, reumatológicas, hepáticas e oncológicas, entre outras), transitam anualmente para serviços de adultos porque completam 18 anos, mas só menos de metade têm acesso a serviços e apoios adequados nesta passagem. ${ }^{3}$

Esta mudança pode ser feita numa lógica de transferência ou de transição.

Transferência implica o encerramento de cuidados no serviço pediátrico e o início de cuidados no serviço de adultos, enquanto que a transição é um processo desenvolvido com propósitos terapêuticos. ${ }^{4} \mathrm{Na}$ primeira situação o jovem chega aos 18 anos e passa para um serviço de adultos, por vezes com um relatório para entregar no novo serviço ou apenas com alta para o Médico de Família. Não existe qualquer comunicação direta entre os dois serviços ou os médicos envolvidos. Na transição, a passagem de um serviço para outro é assumida pelos dois serviços em articulação, com preparação de uma estratégia conjunta para que não haja rotura na continuidade de cuidados. Esta estratégia será mais consistente, se incluir um período de consultas com a participação de médicos dos dois serviços envolvidos (Fig. 1).

Considerar que os dois conceitos são semelhantes, sendo a transferência uma versão menor da transição, produz resultados com impacto negativo. ${ }^{5}$

As dificuldades que persistem nesse processo, na maioria das especialidades médicas, ${ }^{3}$ demonstram que devemos prestar maior atenção à transição, para que não constitua um entrave na continuidade de cuidados, por roturas, hiatos ou interrupções no tratamento.

A transição/processo terapêutico deve incluir a preparação da pessoa envolvida, um período de atendimento conjunto, reuniões de planeamento e partilha de informações clínicas, e ser entendido como um processo de continuidade de cuidados. ${ }^{4}$

Um mau planeamento e implentação deste processo, , pode levar a que jovens de 16 a 20 anos não recebam os cuidados apropriados, e como tal, deixem de estar apoiados por uma rede de cuidados.

McDonagh e Viner ${ }^{6}$ apontaram várias dificuldades que surgem na transição, tais como: falta de treino dos profissionais envolvidos, dificuldades na obtenção dos recursos necessários, má coordenação entre as organizações de saúde e falta de apoio institucional, de planeamento e de especialistas com experiência.

O estabelecimento de normas de orientação clínica que diminuam o impacto da transição influencia positivamente o prognóstico da doença. Devemos avaliar os programas / processos de transição e seu impacto numa base contínua. $^{7}$

Estas normas de orientação clínica podem incluir equipas de transição autónomas dentro do sistema de saúde ${ }^{2}$ e uma abordagem multidisciplinar com envolvimento do paciente e pessoas significativas. A avaliação deve incluir outros parâmetros além da idade, produzindo uma transição partilhada, maior cooperação entre os serviços e en-

\footnotetext{
1. Clínica 1 - Unidade Partilhada. Centro Hospitalar Psiquiátrico de Lisboa. Lisboa. Portugal.

$\triangle$ Autor correspondente: José Salgado. salgadopsi@gmail.com

Recebido: 08 de fevereiro de 2021 - Aceite: 20 de dezembro de 2021 - Online issue published: 02 de março de 2022
}

Copyright $\odot$ Ordem dos Médicos 2022 


\section{Consulta Pediátrica}

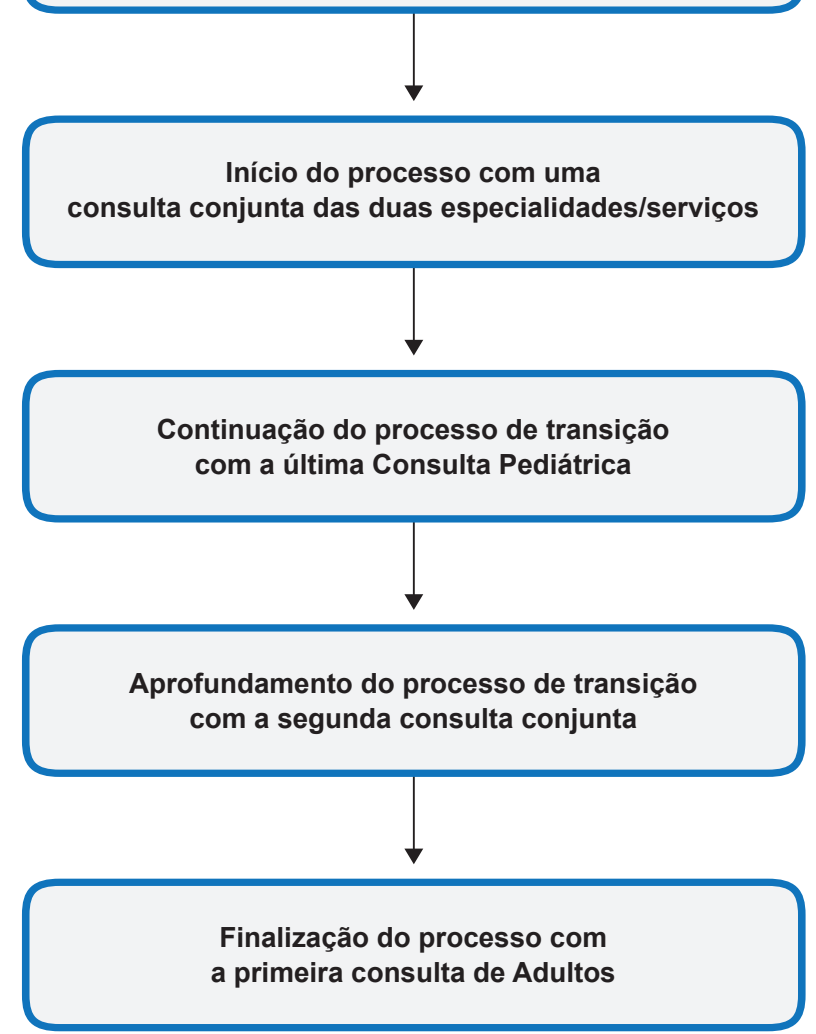

Figura 1 - Fluxograma de colaboração entre serviços no processo de transição

volvimento de profissionais de saúde pediátrica e adulta. É frequente os jovens terem alta dos serviços pediátricos e é o Médico de Família que articula e concretiza a passagem para os serviços de adultos.

Nas últimas décadas, o debate sobre as melhores estratégias para uma transição mais eficiente tem aumentado, mas os resultados não conseguiram superar problemas como a dificuldade de comunicação entre os serviços e a rígida barreira dos 18 anos.

Mesmo quando existem protocolos de transição, o resultado do processo é muitas vezes insatisfatório. ${ }^{8}$ Contudo, isso não diminui a sua importância antes reafirma a necessidade de aperfeiçoamento, porque planeamento e operacionalização são elementos essenciais na organização e prestação dos serviços de saúde.

$\mathrm{O}$ estudo TRACK ${ }^{8}$ analisou as transições em Saúde Mental na região de Londres, envolvendo jovens na 'idade de transição', com patologia psiquiátrica. Os autores concluíram que menos de $10 \%$ fizeram uma transição adequada. Noutras especialidades, embora a taxa de sucesso seja maior, geralmente não chega aos $50 \%,{ }^{3}$ isto é, mais de metade sofre um impacto negativo para a sua doença nessa fase da vida.

É redutor pensar que a transição é um processo que deve ser realizado porque o doente chegou à maioridade, independentemente das condições para tal passagem de testemunho ${ }^{9}$ (cooperação entre os serviços, proximidade geográfica e colaboração da família, etc.).

Como tal, o doente não deve mudar de serviço porque tem 18 anos, mas porque tem condições para o fazer, num processo que pode começar aos 16 ou aos 20, e considerando: maturidade da personalidade, gravidade e consciência da doença e consistência do suporte familiar e social, e capacidade dos serviços pediátricos e de adultos para se envolverem no processo de transição (reconhecendo a vantagem de estratégias de comunicação assertivas e/ou desenvolvendo protocolos para essa mudança).

Existem resistências que podem assumir contornos diversos, tanto por parte dos serviços de adolescentes como de adultos quando a fronteira dos 18 não é rigorosamente cumprida. Antes dos 18, os serviços de adolescentes consideram que são mais capazes de manter o acompanhamento, enquanto que os segundos consideram que não têm experiência suficiente em lidar com adolescentes, podendo ser perigoso para o jovem por não estarem preparados para lidar com esta faixa etária. ${ }^{10}$

Quando atingem a idade adulta, os serviços de adolescentes podem ter relutância em largar os pacientes pelo receio de estes não tolerarem a rotura dos laços terapêuticos estabelecidos e os serviços de adultos pensam que a intervenção deve ser diferente da que tem sido seguida até então. Podem não acautelar, quer uns quer outros, que a abordagem terapêutica evolua com o crescimento do doente, sem solavancos.

Se os serviços funcionarem em continuidade de cuidados, com um mínimo de perturbações, reduz-se o número de jovens que se perdem nesta transição, ${ }^{7}$ e dessa forma o processo será mais pacífico, de acordo com a pessoa específica e não com a idade.

Um compromisso flexível sem perder rigor, poderá traduzir-se num protocolo de seguimento partilhado pelas duas equipas/especialidades não inferior a seis meses, como mostra o fluxograma (Fig. 1), mas não devendo ultrapassar um ano para não se perpetuar. O número de consultas partilhadas poderia variar, dependendo da avaliação conjunta, caso a caso.

Assim, poderia haver uma transição progressiva e 'obrigatória' de uma especialidade para outra, agilizando a comunicação entre equipas e conduzindo ao estabelecimento de pontes. O paciente e sua família não se sentiriam desapoiados pelo serviço que conhecem e seria mais fácil entrar no novo serviço.

Embora possa haver alguma subjetividade no processo de transição, ${ }^{4}$ a simples transferência comporta mais riscos, porque não sabemos como será a chegada do jovem ao novo serviço.

Acreditamos que um processo de transição, discutido e organizado em colaboração, não é a única forma de passar de um serviço pediátrico para um de adultos, mas será aquela que trará maiores benefícios para os pacientes e para a dinâmica dos serviços envolvidos. 


\section{CONFLITOS DE INTERESSE}

O autor declara ter recebido apoios da Janssen Farmacêutica e da Lundbeck para a participação em encontros científicos.

\section{REFERÊNCIAS}

1. Singh S, Tuomainen, H. Transition from child to adult mental health services: needs, barriers, experiences and new models of care. World Psychiatry. 2015;14:358-61.

2. Blum RW, Garell D, Hodgman C, Jorissen T, Okinow N, Orr D, et al. Transition from child-centered to adult health-care systems for adolescents with chronic conditions. J Adolesc Health. 1993;14:570-6.

3. Davis A, Brown R, Taylor J, Epstein R, McPheeters M. Transition care for children with special health care needs. Pediatrics. 2014;134:900-8.

4. Paul M, Ford T, Kramer T, Islam Z, Harley K, Singh SP. Transfers \& transitions between child and adult mental health services. $\mathrm{Br} \mathrm{J}$ Psychiatry. 2013;202:S36-40.

5. Kennedy I. Getting it right for children and young people - overcoming cultural barriers in the NHS so as to meet their needs. London: Great Britain Department of Health; 2010.

6. McDonagh J, Viner R. Lost in transition? Between paediatric and adult services. BMJ. 2006;332:435-6.

7. Stewart D, Freeman A, Law M, Healy H, Burke-Gaffney J, Forhan M,

\section{FONTES DE FINANCIAMENTO}

Este trabalho não recebeu qualquer tipo de suporte financeiro de nenhuma entidade no domínio público ou privado

et al. The best journey to adult life. An evidence-based model and best practice guidelines for the transition to adulthood for youth with disabilities. Ontario: Ontario McMaster University; 2009.

8. Singh S, Paul M, Ford T, Kramer T, Weaver T. Transitions of care from child and adolescent mental health services to adult mental kealth services (TRACK Study): a study of protocols in Greater London. BMC Health Serv Res. 2008;8:135.

9. Penttilä M, Jaaskelainen E, Hirvonen N, Isohanni M, Miettunen J. Duration of untreated psychosis as predictor of long-term outcome in schizophrenia: systematic review and meta-analysis. $\mathrm{Br} \mathrm{J}$ Psychiatry. 2014;205:88-94.

10. Famcnod Dh Partnerships for Children. Transition. Moving on well: a good practice guide for health professionals and their partners on transition planning for young people with complex heath needs of a disability. (2008). [consultado 2022 jan 16]. Disponível em: https:// www.semanticscholar.org/author/Famcnod-Dh-Partnerships-forChildren/84562731. 